\title{
Pathologic Stage IA Gastric Cancer AJCC v8
}

National Cancer Institute

\section{Source}

National Cancer Institute. Pathologic Stage IA Gastric Cancer AJCC v8. NCI Thesaurus.

Code C133656.

Stage IA includes: (T1, N0, M0). T1: Tumor invades the lamina propria, muscularis mucosae, or submucosa. N0: No regional lymph node metastasis. M0: No distant metastasis. (AJCC 8th ed.) 